\title{
A REMARK ON THE NILPOTENCY INDEX OF THE RADICAL OF A GROUP ALGEBRA OF A $p$-SOLVABLE GROUP
}

\author{
by SHIGEO KOSHITANI
}

(Received 6th June 1980)

Let $K$ be a field of characteristic $p>0, G$ a finite $p$-solvable group, $P$ a $p$-Sylow subgroup of $G$ of order $p^{a}, K G$ the group algebra of $G$ over $K$, and $J(K G)$ the Jacobson radical of $K G$. In the present paper we study the nilpotency index $t(G)$ of $J(K G)$, which is the least positive integer $t$ with $J(K G)^{t}=0$. Since $J(E G)=$ $E \otimes_{K} J(K G)$ for any extension field $E$ of $K$ (cf. [7, Proposition 12.11]), we may assume that $K$ is algebraically closed.

D. A. R. Wallace [12] proved that

$$
t(G) \geqq a(p-1)+1 .
$$

There is a problem to determine the structure of $G$ with the property $t(G)=$ $a(p-1)+1$. When $G$ is of $p$-length 1 , by the results of S. A. Jennings [6] and K. Morita [8], $t(G)=a(p-1)+1$ if and only if $P$ is elementary abelian (cf. [10, Corollary 1]). But for $p$-solvable groups $G$ of $p$-length $\geqq 2$ the assertion does not hold in general. Indeed, $K$. Motose and Y. Ninomiya [10] showed that when $p=2$ and $G=S_{4}$ (which denotes the symmetric group of degree 4$), t(G)=4$ though $P$ is dihedral of order 8 . Recently, K. Motose [9] proved that if $p=2, P$ is metacyclic and $G / O_{2^{\prime}}(G) \neq S_{4}$, then $t(G)=$ $a+1$ if and only if $P$ is elementary abelian. The purpose of this paper is to consider the proposition for the case where $p$ is odd. If $p$ is odd and $P$ is metacyclic, then $P$ is a regular $p$-group (cf. [5, III 10.2 Satz (c)]). Y. Tsushima [11] claimed that when $P$ is regular, $t(G)=a(p-1)+1$ if and only if $P$ is elementary abelian. At line 11 of page 37 in [11], he says that since $P$ has exponent $p, G$ is of $p$-length 1 from [4, Theorem A (ii)]. However, Tsushima's assertion is not correct. There exists an example (to be given later) of a $p$-solvable group $G$ of $p$-length $\geqq 2$ such that $P$ has exponent $p$ and so that $P$ is regular. Our main result can be stated as follows: If $p$ is odd and $P$ is metacyclic, then $t(G)=a(p-1)+1$ if and only if $P$ is elementary abelian.

Throughout this paper we use the following notation. We write $O_{p^{\prime}}(G)$ and $O_{p}(G)$ for the maximal normal subgroup of $G$ of order prime to $p$ and the maximal normal $p$-subgroup of $G$, respectively. We define $O_{p^{\prime}, p}(G)$ by $O_{p}\left(G / O_{p^{\prime}}(G)\right)=O_{p^{\prime}, p}(G) / O_{p^{\prime}}(G)$. We write $H \triangleleft G$ if $H$ is a normal subgroup of $G$. For a finite group $Y,|Y|$ and Aut (Y) denote the order of $Y$ and the group of all automorphisms of $Y$, respectively. When $X$ is a subgroup of $G$, we write $N_{G}(X), C_{G}(X)$ and $|G: X|$ for the normaliser of $X$ in $G$, the centraliser of $X$ in $G$ and the index of $X$ in $G$, respectively. If $x_{1}, \ldots, x_{n}$ are in $G$, we write $\left\langle x_{1}, \ldots, x_{n}\right\rangle$ for the subgroup of $G$ generated by $\left\{x_{1}, \ldots, x_{n}\right\}$. When $H$ is a 
subgroup of $G$ and $g \in G$, let $[H, g]=\left\langle h^{-1} g^{-1} h g \mid h \in H\right\rangle$ and $[H, g, g]=[[H, g], g]$. We write $G L(2, p)$ and $S L(2, p)$ for the general linear group and the special linear group, respectively (cf. [3, p. 40]). 2.3].

For an odd prime $p$, we say that $G$ is $p$-stable in the sense of $[\mathbf{1}, p .1104$ Definition

We write $Q d(p)$ for the group defined in $[1, p .1104]$ and $[2, p .32]$. Then $Q d(p)$ is the semi-direct product of $R$ by $S L(2, p)$ with respect to the identity map $S L(2, p) \rightarrow$ $S L(2, p) \subseteq G L(2, p) \simeq$ Aut $(R)$, where $R$ is an elementary abelian group of order $p^{2}$. It is noted that if $p$ is odd then the $p$-Sylow subgroup of $Q d(p)$ is nonabelian of order $p^{3}$ of exponent $p$ (cf. [2, p. 32 and p. 33 Example 11.4]).

To begin with, we state the next two lemmas which are useful for our aim.

Lemma 1. Let $G$ be a finite group and $p$ an odd prime. If the $p-S y l o w$ subgroup of $G$ is of order $p^{3}$ with exponent $p^{2}$, then $G$ is p-stable.

Proof. By [1, Lemma 6.3], it suffices to show that $X / Y \neq Q d(p)$ for any subgroup $X$ of $G$ and any $Y \triangleleft X$ (see [1, p. 1103] for the term "involved"). Assume that $X / Y \simeq$ $Q d(p)$ for some subgroup $X$ of $G$ and some $Y \triangleleft X$. Since the order of the $p$-Sylow subgroup of $Q d(p)$ is $p^{3}$ by [2, p. 32], $p \nmid|G: X|$ and $p \nmid|Y|$. Let $P$ be a $p$-Sylow subgroup of $X$. Then $P$ is a $p$-Sylow subgroup of $G$, so that $P$ has exponent $p^{2}$. On the other hand, $(P Y) / Y$ is a $p$-Sylow subgroup of $X / Y$. Hence $(P Y) / Y$ has exponent $p$ from [2, p. 33 Example 11.4]. This is a contradiction since $(P Y) / Y \simeq P /(P \cap Y) \simeq P$. This completes the proof.

Lemma 2 [3, Theorem 8.1.3]. Let $p$ be an odd prime, and let $G$ be a finite group with a p-Sylow subgroup $P$ such that $O_{p}(G) \neq 1$ and $G$ is p-stable and p-solvable. If $A$ is an abelian normal subgroup of $P$, then $A \subseteq O_{p^{\prime}, p}(G)$.

Proof. Let $H=O_{p^{\prime}, p}(G), \quad Q=P \cap H, \quad N=N_{G}(Q)$ and $C=C_{G}(Q)$. Then $O_{\mathrm{p}}(G) \cdot Q=H \triangleleft G$. Take any $x \in A$. Clearly $x \in N$. Since $A \triangleleft P \supseteq Q,[Q, x] \subseteq A$. Since $A$ is abelian, $[Q, x, x] \subseteq[A, x]=1$, so that $[Q, x, x]=1$. Since $G$ is $p$-stable, $x C \in$ $O_{p}(N / C)$. This shows $(A C) / C \subseteq O_{p}(N / C)$. Since $G$ is $p$-solvable, $C \subseteq H$ by [3, Theorem 6.3.3], so that $C \subseteq H \cap N$. By the Frattini argument [3, Theorem 1.3.7], $G=H N$. Then we have the following epimorphism

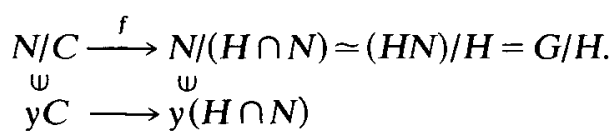

Since $H=O_{p^{\prime}, p}(G), O_{p}(G / H)=1$, so that $O_{p}(N /(H \cap N))=1$. Since $f$ is an epimorphism, $f\left(O_{p}(N / C)\right) \subseteq O_{p}(N /(H \cap N))$. This implies $f((A C) / C)=1$, so that $A \subseteq H \cap N$.

Using these lemmas we can prove the next main result of this paper.

Theorem. Let $p$ be an odd prime, and let $G$ be a finite $p$-solvable group with $a$ metacyclic $p$-Sylow subgroup $P$ of order $p^{a}$. Then $t(G)=a(p-1)+1$ if and only if $P$ is elementary abelian. 
Proof. Assume that $P$ is elementary abelian. By [3, Theorem 6.3.3], $P \subseteq O_{p^{\prime}, p}(G)$. This implies that $G$ is of $p$-length 1 . So that $t(G)=a(p-1)+1$ by [10, Corollary 1$]$.

Suppose $t(G)=a(p-1)+1$. We use induction on $|G|$. Assume $G \neq 1$. Let $H=$ $O_{p^{\prime}}(G)$. By [12, Theorems 2.2 and 3.3], $a(p-1)+1 \leqq t(G / H) \leqq t(G)=a(p-1)+1$. Hence we may assume $H=1$ by induction. Let $R=O_{p}(G)$ and $|R|=p^{b}$, so that $1 \leqq b \leqq a$. Then $a(p-1)+1=t(G) \geqq t(R)+t(G / R)-1$ by [12, Theorem 2.4]. Since $t(R) \geqq b(p-1)+1$ and $t(G / R) \geqq(a-b)(p-1)+1$ by [12, Theorem 3.3], we have $t(R)=$ $b(p-1)+1$. So that $R$ is elementary abelian from [10, Theorem 1]. Since $P$ is metacyclic, $R$ is cyclic of order $p$ or is elementary abelian of order $p^{2}$. Then $C_{G}(R)=R$ by [3, Theorem 6.3 .3$]$, so that

$$
G / R=N_{G}(R) / C_{G}(R) \hookrightarrow \text { Aut }(R) .
$$

If $R$ is cyclic of order $p$, then $p \nmid|G / R|$ by $(*)$, so that $P$ is cyclic of order $p$. Hence we may assume that $R$ is elementary abelian of order $p^{2}$. By [10, Corollary 1], it suffices to show that $G$ is of $p$-length 1 . Suppose that $G$ is of $p$-length $\geqq 2$. Since $\mid$ Aut $(R) \mid=$ $|G L(2, p)|=p(p-1)^{2}(p+1)$ by $[3$, Theorem 2.8 .1$],|P / R|=1$ or $p$ from $\left(^{*}\right)$. This shows that $|P|=p^{2}$ or $p^{3}$. Since $G$ is of $p$-length $\geqq 2, P$ is nonabelian from [3, Theorem 6.3.3]. Hence $|P|=p^{3}$. Since $P$ is metacyclic, we can write

$$
P=M_{3}(p)=\left\langle x, y \mid x^{p}=y^{p^{2}}=1, x^{-1} y x=y^{p+1}\right\rangle
$$

by [3, Theorem 5.5.1]. Then $G$ is $p$-stable by Lemma 1 . Since $\left\langle x, y^{p}\right\rangle \triangleleft P$ and $\langle y\rangle \triangleleft P$ and since $R \neq 1$, we have that $\left\langle x, y^{p}\right\rangle \subseteq R$ and $\langle y\rangle \subseteq R$ by Lemma 2 . Then $x, y \in R$, so that $P=R$. Hence $G$ is of $p$-length 1 , a contradiction. This completes the proof.

Finally we give an example as mentioned in the introduction.

Example. Let $p=3$, and let $R$ be an elementary abelian group of order 9 . Let $G$ be the semi-direct product of $R$ by $S L(2,3)$ with respect to the identity map $S L(2,3) \rightarrow$ $S L(2,3) \subseteq G L(2,3) \simeq$ Aut $(R)$. Then $G \simeq \operatorname{Qd}(3)$ (cf. [1, p. 1104] and [2, p. 32]). Let $R=\langle b, c\rangle$ and $S=S L(2,3)$. For each $x=\left(\begin{array}{cc}s & t \\ u & v\end{array}\right) \in S$, we can write that $x^{-1} b x=b^{s} c^{t}$ and $x^{-1} c x=b^{u} c^{v}$. Let $a=\left(\begin{array}{ll}1 & 1 \\ 0 & 1\end{array}\right) \in S$, then $a$ is of order 3 , so that we can write $P=\langle a, b$, $c\left|a^{3}=b^{3}=c^{3}=1, a^{-1} b a=b c, a^{-1} c a=c, b^{-1} c b=c\right\rangle$, where $P$ is a 3-Sylow subgroup of $G$. Then $P$ has exponent 3 (cf. [2, pp. 32-33] and [3, p. 203]). Let $Q$ be a 2-Sylow subgroup of $S$. Since $Q \triangleleft S$ and since $Q$ is quaternion of order $8, S$ has the unique involution $z=\left(\begin{array}{rr}-1 & 0 \\ 0 & -1\end{array}\right)$ in $Q$. Let $H=O_{3}(G)$. Since $|G|=2^{3} \cdot 3^{3}=216, H=O_{2}(G)$. Since $Q$ is a 2-Sylow subgroup of $G, H \subseteq Q$. Evidently, $H R=H \times R$. If $H \neq 1$, then $z \in H$, so that $z \in C_{G}(R)$, a contradiction. Hence $H=1$. On the other hand, $P$ is not normal in $G$. So that $G$ is of 3-length 2 .

Acknowledgement. The author wishes to express his gratitude to the referee for his kind advice.

\section{REFERENCES}

1. G. Glauberman, A characteristic subgroup of a p-stable group, Canad. J. Math. 20 (1968), 1101-1135. 
2. G. Glauberman, Global and local properties of finite groups, Finite simple groups (edited by M. B. Powell and G. Higman) (Academic Press, New York, 1971), 1-64.

3. D. Gorenstein, Finite groups (Harper \& Row, New York, 1968).

4. P. Hall and G. Higman, On the p-length of $p$-soluble groups and reduction theorems for Burnside's problem, Proc. London Math. Soc. (3) 6 (1956), 1-42.

5. B. Huppert, Endliche Gruppen I (Springer, Berlin, 1967).

6. S. A. Jennings, The structure of the group ring of a $p$-group over a modular field, Trans. Amer. Math. Soc. 50 (1941), 175-185.

7. G. O. Michler, Blocks and centers of group algebras (Lectures on rings and modules, Lecture notes in math. 246, Springer, Berlin, 1972), 429-563.

8. K. Morita, On group rings over a modular field which possess radicals expressible as principal ideals, Science Reports of Tokyo Bunrika Daigaku A4 (1951), 177-194.

9. K. Motose, On the nilpotency index of the radical of a group algebra II, Math. J. Okayama Univ. 22 (1980), 141-143.

10. K. Motose and Y. NinomiYa, On the nilpotency index of the radical of a group algebra, Hokkaido Math. J. 4 (1975), 261-264.

11. Y. Tsushima, Some notes on the radical of a finite group ring II, Osaka J. Math. 16 (1979), 35-38.

12. D. A. R. WALLACE, Lower bounds for the radical of the group algebra of a finite p-soluble group, Proc. Edinburgh Math. Soc. 16 (1968/69), 127-134.

Department of MAthematics

FACULTY OF SCIENCE

Chiba Universit'y

$1-33$, YAYOI-CHO

Chiba-City, 260

JAPAN 